\title{
Adaptive Immunity: The Role of Toll-Like Receptors
}

Yamaguchi $\mathbf{R}^{1}$, Sakamoto $A^{1}$, Yamaguchi $\mathbf{R}^{2}$, Haraguchi $M^{1}$, Narahara $S^{1}$, Sugiuchi $H^{1}$ and Yamaguchi $\mathbf{Y}^{\text {* }}$

${ }^{1}$ Graduate School of Medical Science, Kumamoto Health Science University, Kitaku Izumi-machi 325 Kumamoto 861-5598, J apan

${ }^{2}$ Department of Neuroscience, Graduate School of Medicine and Faculty of Medicine, Kyoto University, Yoshida-konoe-cho Sakyo-ku Kyoto 606-8501, J apan

*Corresponding author: Yamaguchi Yasuo, Graduate School of Medical Science, Kumamoto Health Science University, Kitaku Izumi-machi 325 Kumamoto 8615598, J apan

Received: July 21, 2021; Accepted: August 12, 2021; Published: August 19, 2021

\section{Abstract}

The central mediators of the adaptive immune response are T cells. The clonal expansion of $T$ cells required for adaptive immunity results from the innate immune response, which is triggered by the stimulation of Toll-Like Receptors (TLRs). The adaptive immune response can cause autoimmune diseases, and Th17 cells are known to contribute to several autoimmune diseases. Pathogenic Th17 cells are induced by Interleukin 23 (IL-23) and IL-1 $\beta$. Resiquimod (a TLR7/8 agonist) significantly enhances

IL-23 production by human macrophages, and lipopolysaccharide (a TLR4 agonist) slightly enhances it. Interestingly, IL-23 levels are significantly attenuated after sequential stimulation with lipopolysaccharide and resiquimod, indicating cross-talk between the TLR4 and TLR7/8 signaling pathways. In this review, we discuss the pivotal role of TLRs in triggering innate immunity and inducing adaptive immunity, leading to autoimmune diseases.

Keywords: Adaptive immunity; Innate immunity; TLR4; TLR7/8; IL-23; TNFAIP3

\section{Abbreviations}

ELISA: Enzyme-Linked Immunosorbent Assay; GM-CSF: Granulocyte-Macrophage Colony-Stimulating Factor; IRAK: IL-1 Receptor-Associated Kinase; LPS: Lipopolysaccharide; MAPK: Mitogen-Activated Protein Kinase; NLRP3: NOD-, LRRand Pyrin Domain-Containing Protein 3; NOD: NucleotideBinding Oligomerization Domain-Containing Protein; PAR-2: Protease-Activated Receptor 2; siRNA: small interfering RNA; TAK: Transforming Growth Factor- $\beta$-Activated Kinase; TGF: Transforming Growth Factor; TLR: Toll-Like Receptor; TNFAIP3: Tumor Necrosis Factor $\alpha$-Induced Protein 3

\section{Introduction}

Innate immunity is triggered by Pattern Recognition Receptors (PRRs) expressed on macrophages [1]. These PPPs, which include membrane receptors (Toll-Like Receptors (TLR) 2/4/5) [2], endosomal receptors (TLR3/7/8/9) [3], and a cytosolic receptor (Nucleotide-Binding Oligomerization Domain-Containing Protein $1 / 2, \mathrm{NOD} 1 / 2$ ) [4], activate macrophages and neutrophils in the innate immune response [5]. The innate immune response then leads to the development of adaptive immunity [6]. Unclear is which factors affect induction of the adaptive immune responses after stimulation of the innate immune response. This question is important because adaptive immune responses trigger autoimmune diseases [7].

The key players in the adaptive immune system are lymphocytes, which include $\mathrm{T}$ cells. CD4+ helper $\mathrm{T}$ (Th) cells regulate adaptive immune responses and play a pathogenic role in autoimmune diseases. Th cells differentiate into Th1 and Th2 cells and secrete cytokines, which play a pivotal role in the pathogenesis of autoimmune diseases. Th type 17 (Th17) cells are reported to secrete the cytokine Interleukin 17 (IL-17) and have also been suggested to be closely associated with the development of autoimmune diseases [9], and IL-23, a unique heterodimeric cytokine composed of IL-12p40 and IL-23p19 subunits [8], is required to differentiate and maintain Th17 cells [10].

The innate immune response activated through Toll-Like Receptors (TLRs) may be involved in the initiation and progression of autoimmune diseases. We previously reported that the TLR7/8 agonist resiquimod induced IL-23 produced by human macrophages [11]. This raised the question whether or not the adaptive immune response is also regulated by TLRs. In this review, we discuss the role of TLRs in adaptive immune responses.

\section{Materials and Methods \\ Ethics statement}

The Board of Ethics in Kumamoto Health Science University approved to obtain blood from volunteers in conformity with the declaration of Helsinki after obtaining their informed consent (No. 17046).

\section{Chemicals and reagents}

Recombinant human GM-CSF and Escherichia coli 0111:B4 Lipopolysaccharide (LPS) were purchased from Tocris Bioscience (Bristol, UK) or Sigma-Aldrich (St. Louis, MO), respectively. TLR7/8 agonist, resiquimod, was obtained from ChemScene Chemicals, Monmouth Junction, NJ. Induction of GM-CSF-dependent human macrophages Peripheral Blood Mononuclear Cells (PBMCs) was obtained from heparinized blood samples. PBMCs collected using Lymphoprep gradients (Axis-Shield PoC As, Norway) were suspended with Lymphocyte medium for thawing (BBLYMPH1, ZenBio, Inc. Research Triangle Park, NC). The monocytes were stained with CD14-Phycoerythrin (PE) mouse anti-human monoclonal antibody (Life technologies, Staley Road Grand Island, NY). GM-CSF dependent macrophages were obtained after monocytes stimulated with recombinant human GM-CSF on days 1, 3, and 6 of culture.

Macrophages (on day 9 of culture) were utilized as GM-CSF dependent macrophages in this study.
Austin J Allergy - Volume 7 Issue 1 - 2021

Submit your Manuscript | www.austinpublishinggroup.com

Yamaguchi et al. (C) All rights are reserved
Citation: Yamaguchi R, Sakamoto A, Yamaguchi R, Haraguchi M, Narahara S, Sugiuchi H, et al. Adaptive Immunity: The Role of Toll-Like Receptors. Austin J Allergy. 2021; 7(1): 1038. 


\section{Preparation of whole-cell lysates from cell culture}

Human macrophages (on day 9 of culture) were stimulated with HNE $(5 \mu \mathrm{M})$ or SP $(5 \mu \mathrm{M})$ for 6 hours and culture medium was carefully removed. Mammalian protein extraction reagent $(100 \mu \mathrm{L}$; M-PER, Thermo Fisher Scientific Inc., Waltham, MA) was pipetted into each well, after which the culture plate was gently shaken for 5 minutes.

The lysate was collected and transferred to a microcentrifuge tube for centrifugation at $12,000 \mathrm{~g}$ for 10 minutes. The supernatants were used as a whole-cell lysates in this study.

\section{ELISA for IL-12p40, IL-23 and TNFAIP3}

Macrophages were pretreated with Resiquimod $(5 \mu \mathrm{M})$ and stimulated by LPS (10ng) for 6 hours. The levels of IL-12p40, IL-23 and TNFAIP3 in whole-cell lysates of human macrophages were measured by ELISA (IL-12p40: Abcam, Cambridge, UK, IL-23: Abcam; TNFAIP3: MYBioSource, San Diego) 6 hours after exposure to LPS (10ng) or resiquimod $(5 \mu \mathrm{M})$ for 6 hours. The sensitivity of ELISA for IL-12p40, IL-23 and TNFAIP3 was $20 \mathrm{pg} / \mathrm{mL}, 16.3 \mathrm{pg} / \mathrm{mL}$ and $23.5 \mathrm{pg} / \mathrm{mL}$, respectively.

\section{RNA interferences with TAK-1, TGF $\beta 1 / 2 / 3$, TNFAIP3 siRNA}

Transfection of macrophages with siRNAs for TAK-1 (50nM), TGF $1 / 2 /$ (50nM), TNFAIP3 (50nM) or control siRNA-A (Santa
Cruz Biotechnology, Santa Cruz, CA) was performed day 7-8 of cell culture using Lipofectamine (Life Technologies, Carlsbad, CA). IL12 p40 and IL-23 protein levels in whole-cell lysates or cell-culture supernatants were measured by ELISA.

\section{Statistical analysis}

Results are expressed as the mean (SE). Differences between two groups were analyzed using a $t$-test for independent means, and differences between more than two groups were compared by analysis of variance. When the F ratio was found to be significant, mean values were compared using a post hoc Bonferroni test. $\mathrm{P}<0.05$ was considered to indicate significance in all analyses.

\section{Results}

Resiquimod significantly enhanced IL-23 expression by macrophages, whereas LPS enhanced it only slightly. Furthermore, sequential stimulation of human macrophages with LPS and resiquimod significantly reduced IL-23 levels, as determined by ELISA (Figure 1). LPS upregulated TNFAIP3 expression by human macrophages, as also determined by ELISA (Figure 2). After exposure to resiquimod, small interfering RNA for TGF $\beta 1 / 2 / 3$ or TAK- 1 decreased IL-23 levels (Figure 3). On the other hand, transfection with siRNA for TNFAIP3 significantly upregulated IL-23 (Figure 4).

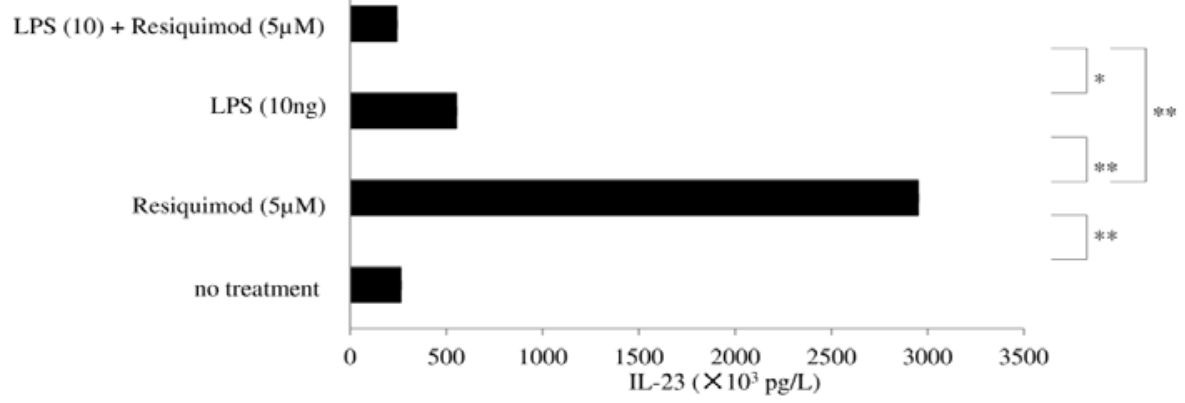

Figure 1: Interleukin 23 production by human macrophages after exposure to resiquimod and lipopolysaccharide.

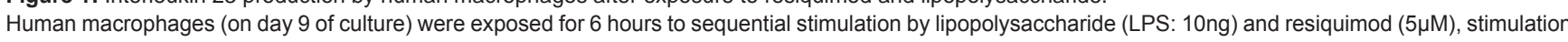
by resiquimod $(5 \mu \mathrm{M})$ or (LPS: 10ng), or no stimulation (no treatment). Data were obtained by using samples from three individuals in each group and represent the mean (SE). ${ }^{* *} \mathrm{P}<0.01 ;{ }^{*} \mathrm{P}<0.05$ (with Bonferroni's correction). Abbreviations: LPS: Lipopolysaccharide.

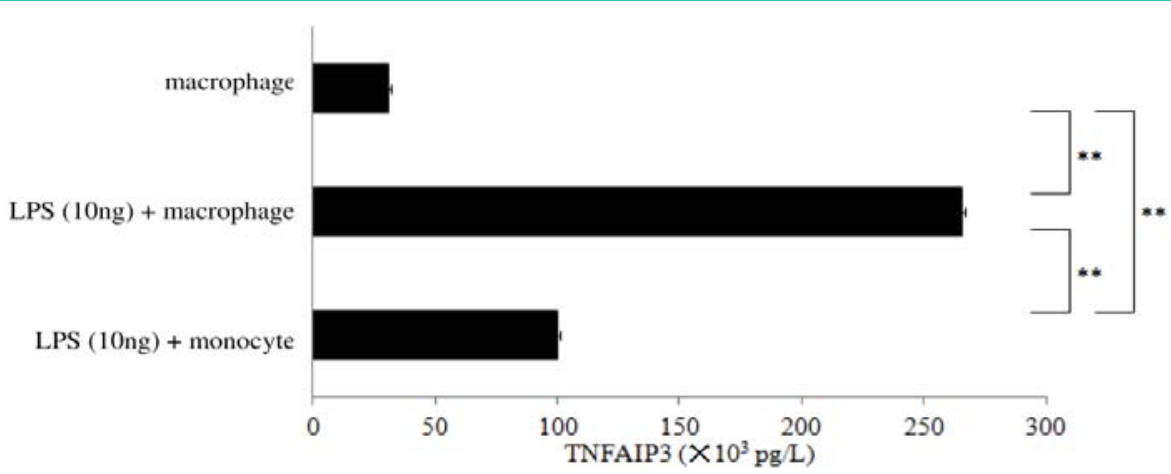

Figure 2: Tumor necrosis factor $\alpha$-induced protein 3 production by human macrophages and monocytes and macrophages after exposure to lipopolysaccharide. Human macrophages and monocytes (on day 9 of culture) were stimulated with lipopolysaccharide (LPS: 10ng) for 6 hours and compared with untreated macrophages. Tumor Necrosis Factor $\alpha$-Induced Protein 3 (TNFAIP3) levels were measured by enzyme-linked immunosorbent assay. Data were obtained by using samples from three individuals in each group. Furthermore, Results are shown as the mean (SE). ${ }^{*} \mathrm{P}<0.01$ (with Bonferroni's correction). Abbreviations: LPS: Lipopolysaccharide; TNFAIP3: Tumor Necrosis Factor $\alpha$-Induced Protein 3. 


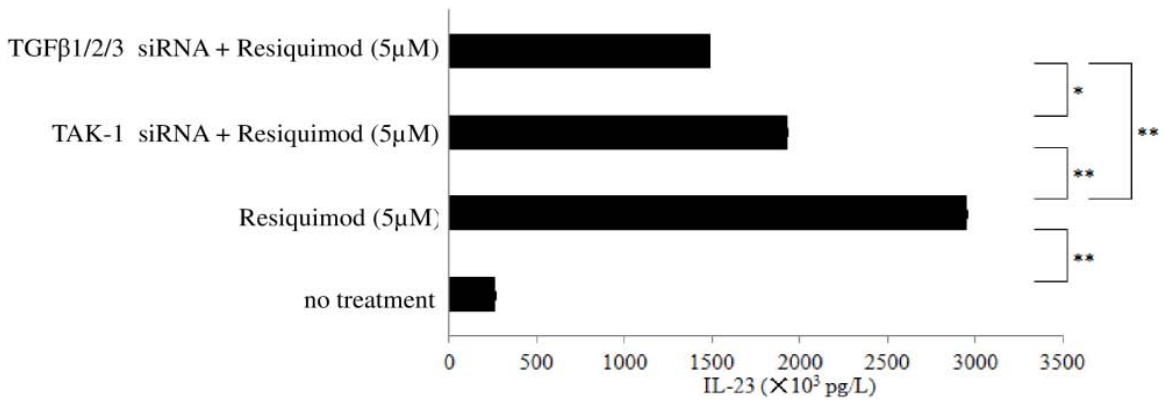

Figure 3: Effect of small interfering RNAs for transforming growth factor $\beta 1 / 2 / 3$, transforming growth factor- $\beta$-activated kinase on interleukin 23 production after exposure to resiquimod.

Human macrophages (on 9 day of culture) transfected with small interfering RNA for transforming growth factor $\beta 1 / 2 / 3$ (TGFB1/2/3) and Transforming Growth Factor- $\beta$-Activated Kinase (TAK-1) were stimulated with resiquimod $(5 \mu \mathrm{M})$ for 6 hours and compared with macrophages stimulated with resiquimod $(5 \mu \mathrm{M})$ for 6 hours without transfection and untreated macrophages. Interleukin 23 levels were then measured by enzyme-linked immunosorbent assay (b). Results are shown as the mean (SE). ${ }^{* *} \mathrm{P}<0.01$; ${ }^{*} \mathrm{P}<0.05$ (with Bonferroni's correction). Abbreviations: IL-23: Interleukin 23; siRNA: small Interfering RNA; TGFß: Transforming Growth Factor ß; TAK: Transforming Growth Factor-ß-Activated Kinase.

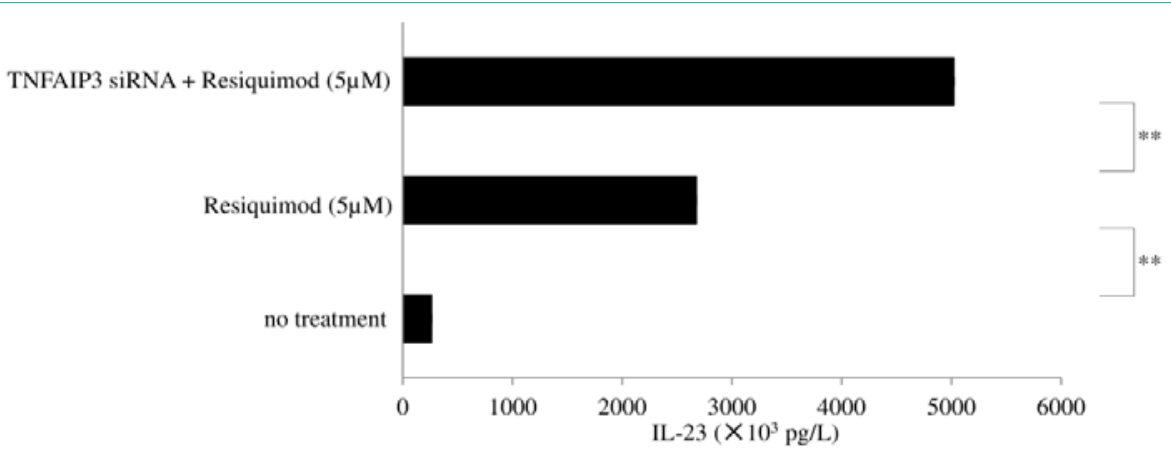

Figure 4: Effect of small interfering RNAs for tumor necrosis factor a-induced protein 3 on interleukin 23 production after exposure to resiquimod. Human macrophages (on 9 day of culture) transfected with small interfering RNA (siRNA) for Tumor Necrosis Factor $\alpha$-Induced Protein 3 (TNFAIP3) were stimulated by resiquimod $(5 \mu \mathrm{M})$ for 6 hours and compared with macrophages stimulated by resiquimod $(5 \mu \mathrm{M})$ without transfection and untreated macrophages. Protein levels of interleukin 23 were then measured by enzyme-linked immunosorbent assay. Data were obtained by using samples from three individuals in each group. Results are shown as the mean (SE). ${ }^{*} \mathrm{P}<0.01$ (with Bonferroni's correction). Abbreviations: IL-23: Interleukin 23; siRNA: small Interfering RNA; TNFAIP3: Tumor Necrosis Factor $\alpha$-Induced Protein 3.

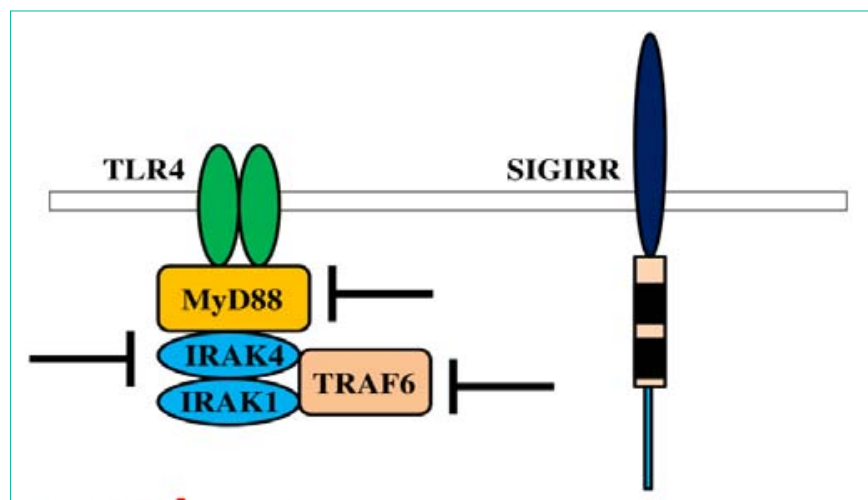

inhibition

Figure 5: Inhibitory effect of expression of toll-like receptor 4 adapto proteins by single Ig IL-1-related receptor. Transmembrane protein Single Ig IL-1-Related Receptor (SIGIRR) is a negative regulator of the TLR4-NF-KB signaling pathway and reduces expression of Myeloid Differentiation Factor 88 (MyD88), IRAK4 and Tumor Necrosis Factor Receptor-Associated Factor 6 (TRAF6). Abbreviations: IRAK: IL-1 Receptor-Associated Kinase; MyD88: Myeloid Differentiation Factor 88; SIGIRR: Single Ig IL-1-Related Receptor TLR: Toll-Like Receptor; TRAF6: Tumor Necrosis Factor ReceptorAssociated Factor 6

\section{Discussion}

\section{Interaction of TLR4 and TLR9 in IL-12p40 production}

The TLR4 agonist LPS stimulates IL-12p40 production. The p35 subunit is known to be constitutively produced, and IL-12 (p35/p40) is known to affect Th1 development [12]. Interestingly, pretreatment of dendritic cells with a TLR9 agonist (CpG oligonucleotides) was reported to additively increase production of IL-12p40 and IL-12p70 [13].

\section{Transactivation of TLR4 by protease-activated receptor 2}

Proteases are released from immune cells and pathogens and activate Protease-Activated Receptors (PARs), which belong to the G-Protein Coupled Receptors (GCPRs). Studies reported that activation of PARs regulates cellular responses of innate and adaptive immune cells [14]. Neutrophils are important innate immune cells that act as phagocytes, release granules and form Neutrophil Extracellular Traps (NETs) during innate immune responses. They release elastase, proteinase, and cathepsin G. Neutrophil elastase activates PAR2, which induces an inflammatory response via a protein kinase C-dependent pathway [15]. A Disintegrin and Metalloproteinase (ADAM) 10/17, one of the major sheddases, is 


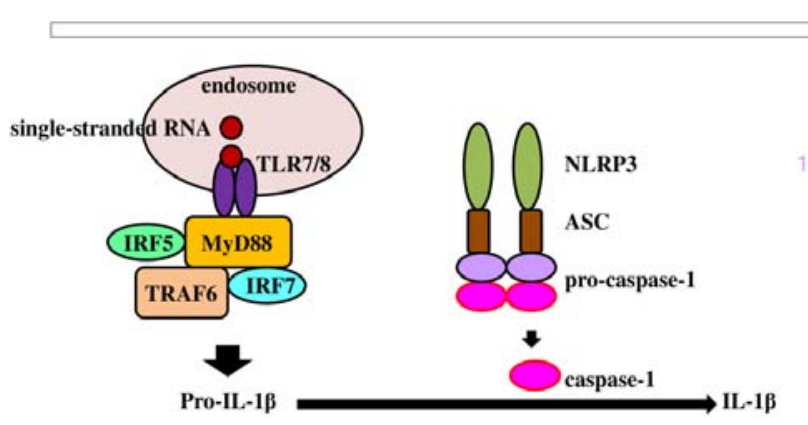

Figure 6: Interleukin $1 \beta$ production by the toll-like receptor $7 / 8 / N O D-$, LRRand pyrin domain-containing protein 3 signaling pathway.

Pro-interleukin- $1 \beta$ is induced by the Toll-Like Receptor (TLR) $7 / 8$ signaling pathway. After activation of NOD-, LRR- and pyrin domain-containing protein 3 , caspase-1-dependent processing of pro-IL-1 $\beta$ promotes IL-1 $\beta$ expression. Abbreviations: ASC: Apoptosis-Associated Speck-Like Protein; IL: Interleukin; IRF: Interferon Regulatory factor; Myd88: Myeloid Differentiation Factor 88; NLPR3: NOD-, LRR- and Pyrin Domain-Containing Protein 3; TLR: Toll-Like Receptor; TRAF6: Tumor Necrosis Factor Receptor-Associated Factor 6.

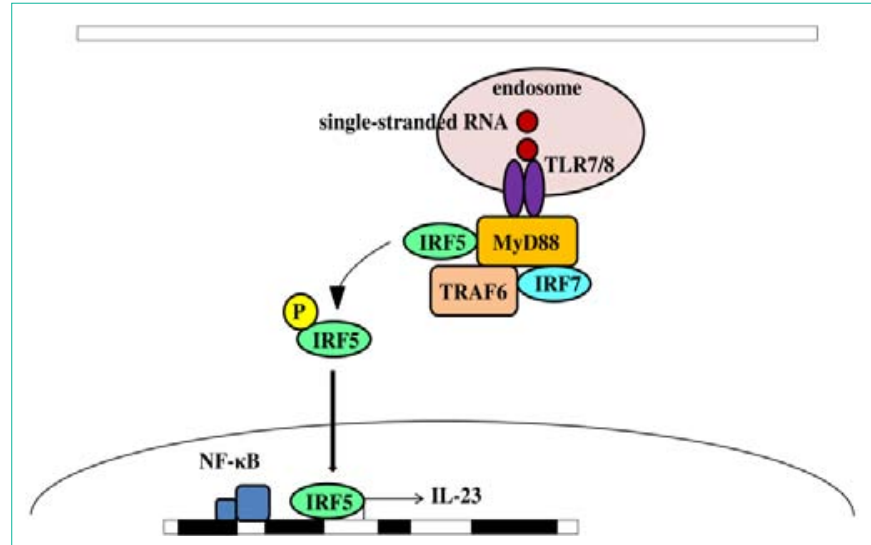

Figure 7: The role of interferon regulatory factor 5 for interleukin 23 expression after stimulation with TLR7/8.

Interferon regulatory factor 5 (IRF5) binds strongly to its target gene, interleukin 23 (IL-23). IRF5 directly activates IL-12p19 expression via the TLR7/8 signaling pathway. Abbreviations: IL: Interleukin; IRF: Interferon Regulatory Factor; Myd88: Myeloid Differentiation Factor 88; NF: Nuclear Factor; P: Phosphate; TLR: Toll-Like Receptor; TRAF6: Tumor Necrosis Factor Receptor-Associated Factor 6.

phosphorylated by protein kinase C [16,17]. Epidermal Growth Factor Receptor (EGFR) is shed by ADAM10/17 [18], and EGFR kinase induces activation of TLR4 [19]. Neutrophil elastase activates PAR2, and PAR2 upregulates Dual Oxidase 2 (DUOX2) [20], which was reported to contribute to ADAM17-mediated shedding of the ectodomain [21]. We reported that neutrophil elastase also induced the transactivation of PAR2/EGFR/TLR4 to increase IL-12p40 production by human macrophages [22].

\section{Cross-talk between TLR4 and TLR7/8 signaling}

p40 is a subunit of both IL-12 (p35/p40) and IL-23 (p19/p40). IL-12 differentiates naïve CD4+ Th cells to Th1 cells via the signal transducer and activator of the transcription Stat 3 and Stat 4 signaling pathways [23]. Th17 cells are known to be involved in the development of autoimmune diseases [9], and, importantly, IL-23 is required to maintain a population of Th17 cells [24]. The TLR4 agonist LPS was reported to stimulate production of IL-12p40 [12]. Interestingly, we

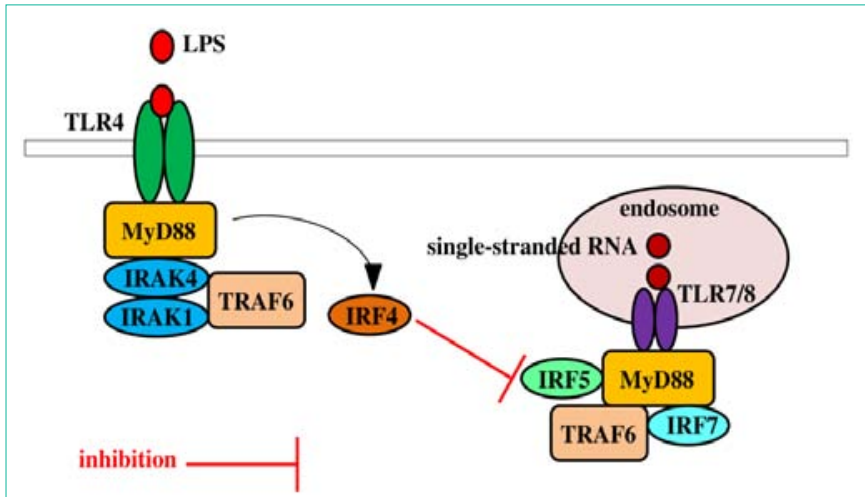

Figure 8: Interferon regulatory factor 4 acts as an antagonist of interferon regulatory factor 5 .

Toll-Like Receptor 4 (TLR4) promotes Interferon Regulatory Factor 4 (IRF4) expression via the Myeloid Differentiation Factor 88 (MyD88) signaling pathway. IRF5 is induced to act as a central mediator of TLR7/8, leading to expression of Interleukin 23 (IL-23). IRF4 binds to MyD88 and selectively competes with IRF5, thus negatively regulating IRF5 activation. Abbreviations: IRAK: IL-1 Receptor-Associated Kinase; IRF: Interferon Regulatory Factor: LPS: Lipopolysaccharide; Myd88: Myeloid Differentiation Factor 88; TLR: Toll-Like Receptor; TRAF6: Tumor Necrosis Factor Receptor-Associated Factor 6 .

found that a higher level of IL-12p40 after LPS stimulation was not associated with production of IL-23.

The TLR7/8 agonist resiquimod induced an increase in IL-23 levels [11], but LPS induced only a slight increase in IL-23. This finding suggests a mechanism that inhibits IL-23 production after TLR4 activation. Transmembrane protein Single Ig IL-1-Related Receptor (SIGIRR) is a negative regulator of the TLR4-NF- $\kappa B$ signaling pathway and reduces expression of Myeloid Differentiation Factor 88 (MyD88), IL-1 Receptor-Associated Kinase 4 (IRAK4), and Tumor Necrosis Factor Receptor-Associated Factor 6 (TRAF6) [25] (Figure 5). Interestingly, we found that RNA interference of SIGIRR significantly increased IL-23 production after stimulation of human macrophages with the TLR7/8 agonist resiquimod. Pro-IL-1 $\beta$ is induced by the TLR7/8 signaling pathway, and the NOD-, LRRand Pyrin Domain-Containing Protein 3 (NLRP3) inflammasome is activated to induce IL-1 $\beta$ expression (Figure 6). Studies reported that SIGIRR inhibits differentiation of Th17 cells by regulating the IL- $1 \beta$ signaling pathway [26] and indicated that SIGIRR is both a negative regulator of TLR 4 and a positive regulator of TLR7/8. These findings led us to the question whether TLR4-related factors regulate IL-23 expression after stimulation of human macrophages with TLR7/8.

Activation of TLR4 promotes IRF4 expression in macrophages [27]. Interferon Regulatory Factor 5 (IRF5) directly activates IL12 p19 expression [28] and was reported to be a pivotal mediator of the TLR7 signaling pathway [29], which produces IL-23 (Figure 7). Studies showed that IRF4 binds to both IRF5 and its target genes. Thus, IRF4 induced by TLR4 activation inhibits IRF5 expression. Vice versa, IRF4 deficiency leads to enhanced expression of IRF5 [30]. IRF4 may act as an antagonist of IRF5 (Figure 8). In addition, SIGIRR attenuates expression of MyD88 [25], and MyD88 signaling induces IRF4 after activation of TLR4. Therefore, reduction of IRF4 levels by SIGIRR means that IRF4 cannot effectively compete with IRF5. Consequently, IL-23 production is increased via the IRF5 signaling pathway (Figure 9). 


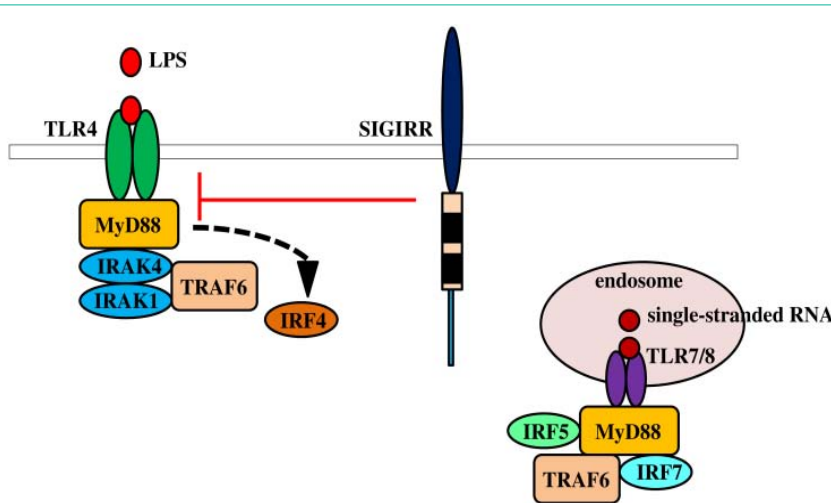

Figure 9: The effect of single Ig IL-1-related receptor on interferon regulatory factor 4 expression.

Interferon Regulatory Factor 4 (IRF4) is induced by Myeloid Differentiation Factor 88 (MyD88) signaling after activation of Toll-Like Receptor 4 (TLR4). Single Ig IL-1-Related Receptor (SIGIRR) inhibits MyD88 expression, which reduces IRF4. This effect indicates that SIGIRR promotes IRF5 signaling by inhibiting IRF4 expression. Abbreviations: IRAK: IL-1 Receptor-Associated Kinase; IRF: Interferon Regulatory Factor; LPS: Lipopolysaccharide; MyD88: Myeloid Differentiation Factor 88; TLR: Toll-Like Receptor; TRAF6: Tumor Necrosis Factor Receptor-Associated Factor 6.

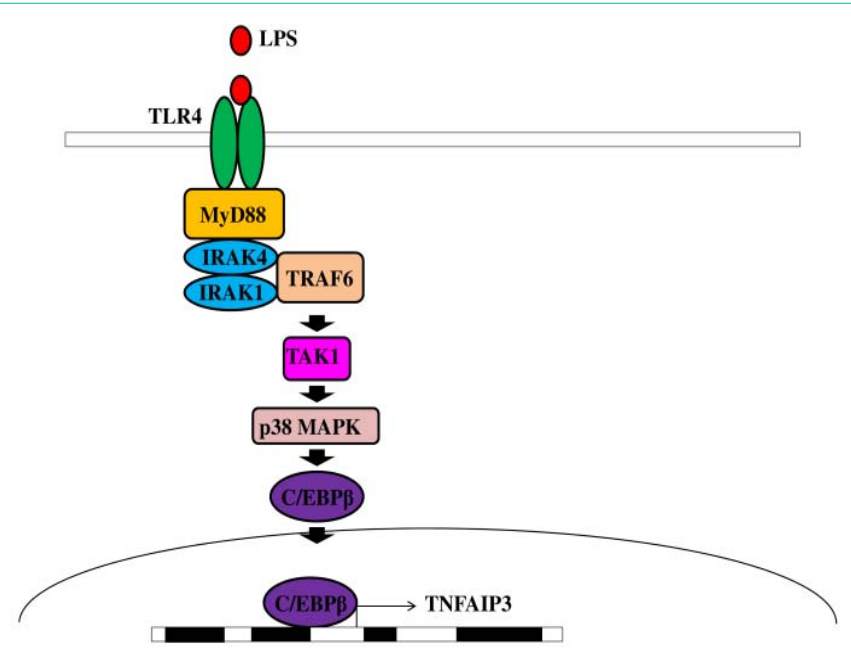

Figure 10: Signaling pathway for expression of tumor necrosis factor a-induced protein 3 after toll-like receptor 4 activation.

Tumor necrosis factor receptor-associated factor 6 ubiquitin ligase activates transforming growth factor- $\beta$-activated kinase, which promotes the p38 Mitogen-Activated Protein Kinase (p38MAPK) signaling pathway. Tumor Necrosis Factor $\alpha$-Induced Protein 3 (TNFAIP3) expression is induced by the p38MAPK/CCAAT-enhancer-binding protein signaling pathway. Abbreviations: C/EBPB: CCAAT-Enhancer-Binding Protein; IRAK: IL-1 Receptor-Associated Kinase; LPS: Lipopolysaccharide; MAPK: MitogenActivated Protein Kinase; TAK: Transforming Growth Factor- $\beta$-Activated Kinase; TNFAIP3: Tumor Necrosis Factor Alpha-Induced Protein 3; TLR: TollLike Receptor; TRAF6: Tumor Necrosis Factor Receptor-Associated Factor 6

\section{The role of TNFAIP3 in regulating IL-23 production}

IL-23 production was significantly upregulated by the TLR7/8 agonist resiquimod but only slightly upregulated by the TLR4 agonist LPS. Interestingly, IL-23 levels were significantly attenuated after sequential stimulation with LPS and resiquimod.

TLR4-related factors induced by LPS may regulate IL-23

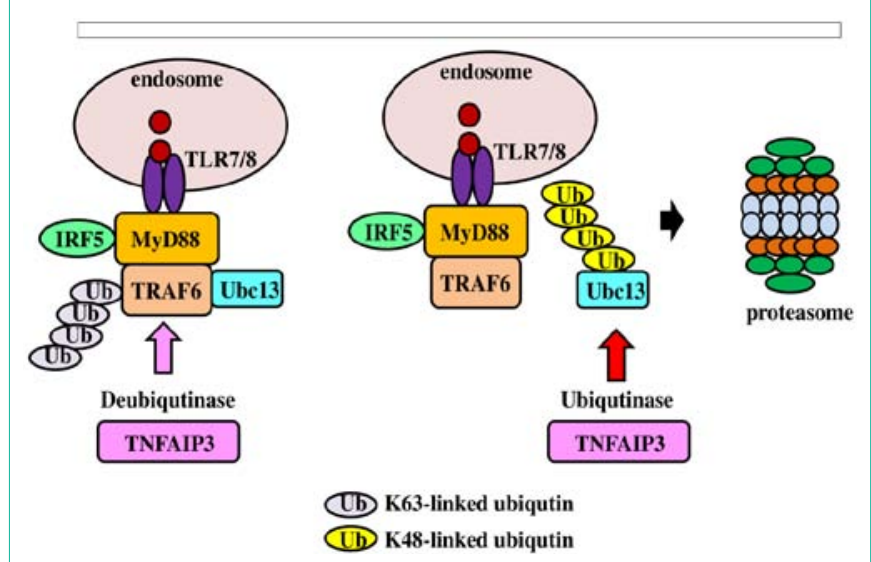

Figure 11: Dual role of ubiquitination and deubiquitination in cellular signals by tumor necrosis factor $\alpha$-induced protein 3 .

Tumor Necrosis Factor Receptor-Associated Factor 6 (TRAF6) acts as an adaptor protein of Toll-Like Receptor 7/8 (TLR7/8). TRAF6, an E3 ubiquitin ligase, promotes $\mathrm{K} 63$-linked polyubiquitination, activates IKB kinase and mediates the activation of NF-kB. Ubiquitin-conjugating enzyme 13 (Ubc13) synthesizes K63-linked ubiquitin chains for TRAF6. Tumor Necrosis Factor a-Induced Protein 3 (TNFAIP3) acts as a deubiquitinase to interact with Ubc13 and cleaves the binding of TRAF6 to Ubc13. Then, TNFAIP3 functions as an ubiquitinase in K48-linked ubiquitination to degrade Ubc13. Abbreviations: IRF: Interferon Regulatory Factor; MyD88: Myeloid Differentiation Factor 88; TNFAIP3: Tumor Necrosis Factor $\alpha$-Induced Protein 3; TLR: Toll-Like Receptor; TRAF6: Tumor Necrosis Factor Receptor-Associated Factor 6; Ub: Ubiquitin; Ubc13: Ubiquitin-Conjugating Enzyme.

expression via TLR7/8 signaling. LPS significantly enhanced TNFAIP3, and studies reported that expression of TNFAIP3 induced by LPS is regulated by both NF- $\mathrm{BB}$ and the p38-dependent C/EBP $\beta$ signaling pathway (Figure 10) [31]. Transforming Growth Factor Beta (TGF- $\beta$ ) [32], IL-6 [33], IL-21 [34], and IL-23 [35] contribute to Th17 formation. TNFAIP3 also plays a role in regulating the IL-23 production triggered by resiquimod. TNFAIP3 has both E3 ubiquitin ligase and deubiquitinase activity and negatively regulates the TGF- $\beta$ signaling pathway [36]. IL-23p19 was reported to be induced by IL- $1 \beta$ [37], and TGF- $\beta$-Activated Kinase 1 (TAK1) is a downstream mediator of IL-1 $\beta$ [38] and a major mediator of NF- $\kappa B$ [39]. Activation of TAK-1 is promoted by Ubiquitin-conjugating enzyme 13 (Ubc13) [40]. TLR7/8 contains an adaptor molecule of TRAF6, which acts as an E3 ubiquitin ligase and promotes K63linked polyubiquitination, enhancing TLR7/8 agonist-mediated signaling. Ubiquitin-conjugating enzyme E2 13 (Ubc13) synthesizes K63-linked ubiquitin chains for TRAF6 [41]. TNFAIP3 contains two domains of ubiquitinase and deubiquitinase, has both E3 ubiquitin ligase and deubiquitinase activity and negatively regulates the TGF- $\beta$ signaling pathway $[36,42]$.

TNFAIP3 acts as a deubiquitinase to interact with Ubc13 and cleave the binding of TRAF6 to Ubc13 [43]. It also enhances the conjugation of Ubc13 to K48-linked ubiquitin chains and promotes the proteasomal degradation of Ubc13 [44]. Furthermore, TNFAIP3 moderates TRAF6 activity by Ubc13 [45] (Figure 11).

\section{Conclusion}

Adaptive immunity is triggered by activation of TLRs, which induce innate immunity. Activation of TLR4 promotes TNFAIP3 generation by human macrophages. TNFAIP3 has both ubiquitinase 
and deubiquitinase activity and regulates the TLR7/8 signaling pathway to enhance IL-23 production. IL-23 maintains Th17 cells and is involved in the development of autoimmune diseases through adaptive immune responses. Adaptive immunity is controlled by cross-talk between TLRs activated by the innate immune system.

\section{Declaration}

Consent for publication: I understand that the text and any pictures published in the article will be freely available on the internet and may be seen by the general public.

Acknowledgement: This study was supported by a Kumamoto Health Science University special fellowship grant (No. 2018-C-02).

\section{References}

1. Amarante-Mendes GP, Adjemian S, Branco LM, Zanetti LC, Weinlich R Bortoluci KR. Pattern Recognition Receptors and the Host Cell Death Molecular Machinery. Front Immunol. 2018; 9: 2379.

2. Hayashi F, Means TK, Luster AD. Toll-like receptors stimulate human neutrophil Function. Blood. 2003; 102: 2660-2669.

3. Patra MC, Achek A, Kim GY, Panneerselvam S, Shin HJ, Baek WY, et al. A Novel Small-Molecule Inhibitor of Endosomal TLRs Reduces Inflammation and Alleviates Autoimmune Disease Symptoms in Murine Models. Cells. 2020; 9: 1648.

4. Vlacil AK, Vollmeister E, Bertrams W, Schoesser F, Oberoi R, Schuett J, et al. Identification of microRNAs involved in NOD-dependent induction of pro-inflammatory genes in pulmonary endothelial cells. PLoS One. 2020; 15: e0228764.

5. Mukhopadhyay S, Plüddemann A, Gordon S. Macrophage pattern recognition receptors in immunity, homeostasis and self-tolerance. Adv Exp Med Biol. 2009; 653: 1-14.

6. Iwasaki A, Medzhitov R. Control of adaptive immunity by the innate immune system. Nat Immunol. 2015; 16: 343-353.

7. Weissert R. Adaptive Immunity Is the Key to the Understanding of Autoimmune and Paraneoplastic Inflammatory Central Nervous System Disorders. Front Immunol. 2017; 8: 336.

8. Yasuda K, Takeuchi Y, Hirota K. The Pathogenicity of Th17 Cells in Autoimmune Diseases. Semin Immunopathol. 2019; 41: 283-297.

9. Parham C, Chirica M, Timans J, Vaisberg E, Travis M, Cheung J, et al. A Receptor for the Heterodimeric Cytokine IL-23 Is Composed of IL-12Rbeta1 and a Novel Cytokine Receptor Subunit, IL-23R. J Immunol. 2002; 168: 56995708.

10. Stritesky GL, Yeh N, Kaplan MH. IL-23 Promotes Maintenance but Not Commitment to the Th17 Lineage. J Immunol. 2008; 181: 5948-5955.

11. Yamaguchi $R$, Sakamoto $A$, Yamamoto $T$, Narahara $S$, Sugiuchi $H$, Yamaguch $Y$. Differential regulation of IL-23 production in M1 macrophages by TIR8/SIGIRR through TLR4- or TLR7/8-mediated signaling. Cytokine. 2017; 99: 310-315.

12. Du C, Sriram S. Mechanism of inhibition of LPS-induced IL-12p40 production by IL-10 and TGF-beta in ANA-1 cells. J Leukoc Biol. 1998; 64: 92-97.

13. Theiner G, Rössner S, Dalpke A, Bode K, Berger T, Gessner A, et al. TLR9 cooperates with TLR4 to increase IL-12 release by murine dendritic cells. Mol Immunol. 2008; 45: 244-252.

14. Shpacovitch V, Feld M, Hollenberg MD, Luger TA, Steinhoff M. Role of protease-activated receptors in inflammatory responses, innate and adaptive immunity. J Leukoc Biol. 2008; 83: 1309-1322.

15. Gatti R, Andre E, Amadesi S, Dinh TQ, Fischer A, Bunnett NW, et al. Protease-activated receptor-2 activation exaggerates TRPV1-mediated cough in guinea pigs. J Appl Physiol. 2006; 101: 506-511

16. Cissé MA, Louis K, Braun U, Mari B, Leitges M, Slack BE, et al. Isoform- specific contribution of protein kinase $\mathrm{C}$ to prion processing. Mol Cell Neurosci. 2008; 39: 400-410.

17. Kveiborg M, Instrell R, Rowlands C, Howell M, Parker PJ. PKCa and PKC regulate ADAM17-mediated ectodomain shedding of heparin binding-EGF through separate pathways. PLoS One. 2011; 6: e17168.

18. Sahin U, Weskamp G, Kelly K, Zhou H-M, Higashiyama S, Peschon J, et al. Distinct roles for ADAM10 and ADAM17 in ectodomain shedding of six EGFR ligands. J Cell Biol. 2004; 164: 769-779.

19. Chattopadhyay S, Veleeparambil M, Poddar D, Abdulkhalek S, Bandyopadhyay SK, Fensterl V, et al. EGFR kinase activity is required for TLR4 signaling and the septic shock response. EMBO Rep. 2015; 16: 15351547.

20. Nadeem A, Alharbi NO, Vliagoftis H, Tyagi M, Ahmad SF, Sayed-Ahmed MM. Proteinase activated receptor-2-mediated dual oxidase-2 up-regulation is involved in enhanced airway reactivity and inflammation in a mouse model of allergic asthma. Immunology. 2015; 145: 391-403.

21. Scheller J, Chalaris A, Garbers C, Rose-John S. ADAM17: a molecular switch to control inflammation and tissue regeneration. Trends Immunol. 2011; 32: 380-387.

22. Yamaguchi R, Yamamoto $T$, Sakamoto A, Narahara $S$, Sugiuchi $H$, Yamaguchi Y. Neutrophil elastase enhances IL-12p40 production by lipopolysaccharide-stimulated macrophages via transactivation of the PAR-2 EGFR/TLR4 signaling pathway. Blood Cells Mol Dis. 2016; 59: 1-7.

23. Charles E Egwuagu. STAT3 in CD4+ T helper Cell differentiation and Inflammatory Disease. Cytokine. 2009; 47: 149-156.

24. Yeh N, Kaplan MH. IL-23 promotes maintenance but not commitment to the Th17 lineage. 2008; 181: 5948-5955.

25. Qin J, Qian Y, Yao J, Grace C, Li X. SIGIRR inhibits interleukin-1 receptorand toll-like receptor 4-mediated signaling through different mechanisms. J Biol Chem. 2005; 280: 25233-25241.

26. Gulen MF, Kang Z, Bulek K, Youzhong W, Kim TW, Chen Y, et al. The receptor SIGIRR suppresses Th17 cell proliferation via inhibition of the interleukin-1 receptor pathway and mTOR kinase activation. Immunity. 2010; 32: 54-66.

27. Eguchi J, Kong X, Tenta M, Wang X, Kang S, Rosen ED. Interferon regulatory factor 4 regulates obesity-induced inflammation through regulation of adipose tissue macrophage polarization. Diabetes. 2013; 62: 3394-3403.

28. Krausgruber T, Blazek K, Smallie T, Alzabin S, Lockstone $\mathrm{H}$, Sahgal N, et al. IRF5 promotes inflammatory macrophage polarization and $\mathrm{TH} 1-\mathrm{TH} 17$ responses. Nat Immuno. 2011; 12: 231-238.

29. Schoenemeyer A, Barnes BJ, Mancl ME, Latz E, Goutagny N, Pitha PM, et al. The interferon regulatory factor, IRF5, is a central mediator of toll-like receptor 7 signaling. J Biol Chem. 2005; 280: 17005-17012.

30. Xu D, Meyer F, Ehlers E, Blasnitz L, Zhang L. Interferon Regulatory Factor 4 (IRF-4) targets IRF-5 to regulate Epstein-Barr virus transformation. J Biol Chem. 2011; 286: 18261-18267.

31. Lai T-Y, Wu S-D, Tsai M-H, Chuang EY, Chuang L-L, Hsu L-C, et al. Transcription of Tnfaip3 is regulated by NF-kB and p38 via C/EBPB in activated macrophages. PLoS One. 2013; 8: e73153.

32. Hatton RD. TGF- $\beta$ in Th17 cell development: the truth is out there. Immunity. 2011; 34: 288-290.

33. Kimura A, Kishimoto T. IL-6: regulator of Treg/Th17 balance. Eur J Immunol. 2010; 40: 1830-1835.

34. Wei L, Laurence A, Elias KM, O'Shea JJ. IL-21 is produced by Th17 cells and drives IL-17 production in a STAT3-dependent manner. J Biol Chem. 2007; 282: 34605-36410.

35. Bunte K, Beikler T. Th17 Cells and the IL-23/IL-17 Axis in the Pathogenesis of Periodontitis and Immune-Mediated Inflammatory Diseases. Int J Mol Sci. 2019; 20: 3394.

36. Long Y, Chen W, Du Q, Zuo X, Zhu H. Ubiquitination in Scleroderma Fibrosis 
and Its Treatment. Front Immunol. 2018; 9: 2383.

37. Liu F-L, Chen C-H, Chu S-J, Chen J-H, Lai J-H, Sytwu H-K, et al. Interleukin (IL)-23 p19 expression induced by IL-1beta in human fibroblast-like synoviocytes with rheumatoid arthritis via active nuclear factor-kappaB and AP-1 dependent pathway. Rheumatology. 2007; 46: 1266-1273.

38. Jurida L, Soelch J, Bartkuhn M, Handschick K, Müller H, Newel D, et al. The Activation of IL-1-Induced Enhancers Depends on TAK1 Kinase Activity and NF-kB p65. Cell Rep. 2015; 10: 726-739.

39. Song Z, Wei W, Xiao W, Al-Saleem ED, Nejati R, Chen L, et al. Essential role of the linear ubiquitin chain assembly complex and TAK1 kinase in A20 mutant Hodgkin lymphoma. Proc Natl Acad Sci USA. 2020; 117: 2898028991

40. Wu X, Zhang W, Font-Bugada J, Palmer T, Alexander S Hamil, Subhra K Biswas, et al. Ubiquitin-conjugating enzyme Ubc13 controls breast cancer metastasis through a TAK1-p38 MAP kinase cascade. Proc Natl Acad Sci USA. 2014; 111: 13870-13875.
41. Branigan E, Plechanovová A, Jaffray EG, Naismith JH, Hay RT. Structural basis for the RING-catalyzed synthesis of K63-linked ubiquitin chains. Nat Struct Mol Biol. 2015; 22: 597-602.

42. Wertz IE, O'Rourke KM, Zhou H, Eby M, Aravind L, Seshagiri S, et al. Deubiquitination and ubiquitin ligase domains of A20 downregulate NF-kappaB signalling. Nature. 2004; 430: 694-699.

43. Biswas R, Bagchi A. Inhibition of TRAF6-Ubc13 interaction in NFkB inflammatory pathway by analyzing the hotspot amino acid residues and protein-protein interactions using molecular docking simulations. Comput Biol Chem. 2017; 70: 116-124

44. Hodge CD, Spyracopoulos L, Glover JNM. Ubc13: the Lys63 ubiquitin chain building machine. Oncotarget. 2016; 7: 64471-64504.

45. Fukushima T, Matsuzawa S, Kress CL, Bruey JM, Krajewska M, Lefebvre $\mathrm{S}$, et al. Ubiquitin-conjugating enzyme Ubc13 is a critical component of TNF Receptor-Associated Factor (TRAF)-mediated inflammatory responses. Proc Natl Acad Sci USA. 2007; 104: 6371-6376. 